\title{
Qualitative Characterization and Use of Prior Information
}

\author{
Martin Eriksson and Stefan Carlsson \\ Royal Institute of Technology, (KTH) \\ Dept. of Numerical Analysis and Computing Science, \\ 10044 Stockholm, Sweden \\ \{eriksson, stefanc\}@nada.kth.se \\ http: //www. nada.kth. se
}

\begin{abstract}
The use of prior information by learning from training data is used increasingly in image analysis and computer vision. The high dimensionality of the parameter spaces and the complexity of the probability distributions however often makes the exact learning of priors an impossible problem, requiring an excessive amount of training data that is seldom realizable in practise. In this paper we propose a weaker form of prior estimation which tries to learn the boundaries of impossible events from examples. This is equivalent to estimating the support of the prior distribution or the manifold of possible events. The idea is to model the set of possible events by algebraic inequalities. Learning proceeds by selecting those inequalities that show a consistent sign when applied to the training data set. The manifold of possible events estimated in this way will in general represent the qualitative properties of the events. We give example of this in the problems of restoration of handwritten characters and automatically tracked body locations
\end{abstract}

\section{Introduction}

Bayesian inference is a methodology that is used frequently in computer vision problem today $[3-5,7,8]$. It's popularity can be partly explained by the fact that it provides a theoretically well founded approach for recognition, restoration, reconstruction etc. given real noisy contaminated data. A key ingredient in Bayesian Inference is the use of prior information. The use of priors allows you to always come up with a result, no matter how bad your data is. For extremely poor data, reliance on priors becomes essential.Very often one is faced with learning priors from insufficient training sets that will bias the result towards the individual exemplars in the set.

In these cases we have to ask for less complete characterizations of the priors. One such more crude characterization is the qualitative nature of the data that we are analyzing. As an example, suppose we want to estimate the shape of a planar curve from a set of noisy points. A qualitative characterization of priors in this cases could just mean that we are considering the class of convex curves. If we let the curve be represented by the set of points $p_{1} \ldots p_{n}$ for which we have noisy measurements, $p_{1}^{\prime} \ldots p_{n}^{\prime}$, the convexity constraint can be expressed as algebraic inequalities for sets of three points at a 


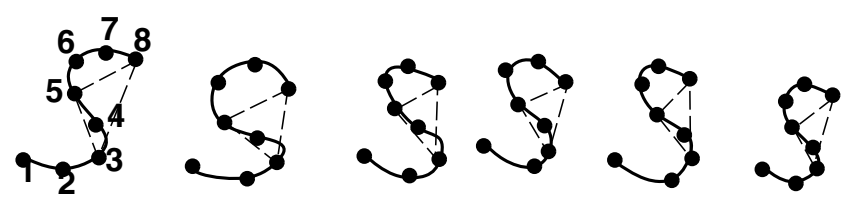

Fig. 1. Examples of handwritten character. Points 3,5 , and 8 is an example of a triplet that has a consistent sign of orientation throughout the set of characters

time. A more complex example could involve shapes like the letter "S" which can be divided into consecutive convex - concave parts. These can still be represented by the orientation of triplets of points computed as the sign of the determinant.

$$
C\left(p_{i}, p_{j}, p_{k}\right)=\left|\begin{array}{ccc}
p_{i} & p_{j} & p_{k} \\
1 & 1 & 1
\end{array}\right|>0
$$

Some triplets of points in a letter "S" will consistently have the same sign while others will have randomly varying signs (fig. 1). The idea is to use the determinant triplets with consistent sign over the training data set as a prior representation the letter " $\mathrm{S}$ ". If we denote by $C_{i}^{*}$ those determinants, we define the set :

$$
M=\left\{p_{1} \ldots p_{n} \mid C_{i}^{*}\left(p_{1} \ldots p_{n}\right)>0 \forall i\right\}
$$

as the constraint manifold for the letter " $\mathrm{S}$ " The Bayesian inference problem can be formulated as that of finding the convex set $p_{1} \ldots p_{n}$ that maximizes the posterior:

$$
\max _{p_{1} \ldots p_{n} \in M} P\left(p_{1}^{\prime} \ldots p_{n}^{\prime} \mid p_{1} \ldots p_{n}\right)
$$

The basic idea can be seen as that of identifying completely impossible events and prevent these from showing up in the results. This is a very weak characterization of the prior information compared to a complete estimation of the pdf and it will consequently allow the measured data more weight in the final estimate. No matter how poor our data are however, we will never get unrealistic results in the final estimation provided we have estimated the constraint manifold.

\subsection{Previous Work}

Bayesian inference has been a common tool in many computer vision applications. For example, Leventon and Freeman [7] approached the problem of human motion reconstruction by using a set of motion capture data to form a set of basis function for the Bayesian prior. Pioneering work in defining dynamic manifolds for human motion was done by Brand [4] who reconstructed human body poses from 2D shadows, assuming the correct reconstruction to reside on a manifold in configuration space. Gomes and Mojsilovic [6] presented a variational approach to define a manifold, given a set of sample points. In our work, we define the dynamic manifold in terms of qualitative properties of point-sets. Aichholzer et. al. [2] presents the concept of qualitatively equivalent point-sets, which is the fundament in the work presented in this paper. 


\section{Learning and Representing the Constraint Manifold}

In order to represent the constraints, we use a variation of the concept of order-types[1]. In principle, any point-set of a certain size has a finite number of qualitatively equivalent configurations. The manifold of allowable configurations can then be regarded as a subset of all possible configurations. The constraint manifold, $M$, can be represented in a constraints table. Figure 2 shows 14 qualitatively different configurations of a set of four points. The table to the left shows how these configurations can be coded. A constraints table, representing a certain class of point-sets, is a subset of this

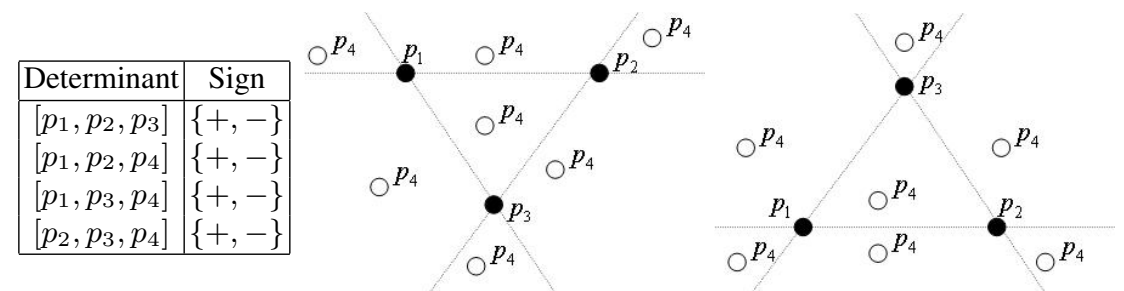

Fig. 2. There are 14 qualitatively distinct configuration of a pointset of cardinality four. Seven of these are shown in the left picture and seven are shown in the right. The table to the left shows how each configuration can be represented.

table, consisting of the triplets posing the qualitative constraints of the class. In order to identify which triplets should go into the constraints-table, a number of training-sets are required. Every point triplet whose determinant has the same sign in every training shape is added to the constraints-table, together with the sign of that particular determinant. For illustration, figure 3 shows an example of a constraints-table with respect to four training-sets. If only one training-set is used, a point-set must have exactly the same determinant table as this only training-set in order to belong to the class. As new training point-sets are added, constraints are removed from the table, and the class gets less constrained. In fig. 4 the effect of adding training-shapes is shown. In this case, the shapes come from the class of ampersands. The graph shows the number of constraining triplets after each of the ampersand-shapes is added to the training-set. After the first two shapes, the number remains rather constant. As the last three shapes are added, the number of constraints drops quickly, due to the strong perturbations of those shapes. In particular, as the last training-shape is added (the mirrored shape) only 10 constraints were left.

\section{Bayesian Inference - Smoothing}

As mentioned in the introduction, given a perturbed point-set $X^{\prime}=p_{1}^{\prime}, p_{2}^{\prime}, \ldots, p_{n}^{\prime}$ we want to find the the point-set $X=p_{1}, p_{2}, \ldots, p_{n}$ maximizing equation 2 . This is done by iteratively refine an initial estimate. The procedure for doing this is outlined below: 


\begin{tabular}{|c|c|c|c|c|c|}
\hline Determinant & Sequence 1 & Sequence 2 & Sequence3 & Sequence 4 & Resulting Constraint \\
\hline$\left[p_{1} p_{2} p_{3}\right]$ & + & + & + & + & + \\
{$\left[p_{1} p_{2} p_{4}\right]$} & + & + & + & + & + \\
{$\left[p_{1} p_{2} p_{6}\right]$} & - & - & - & - & - \\
$\ldots$ & $\ldots$ & $\ldots$ & $\ldots$ & $\ldots$ & $\ldots$ \\
{$\left[p_{n-2} p_{n-1} p_{n}\right]$} & - & - & - & - & - \\
\hline
\end{tabular}

Fig. 3. The constraint table computed using four training sequences. In the last column, the determinant sign of the constraining triplets is shown. Triplets not listed in the table are not constraining.

1. Select an initial $X_{0}$ s.t. $X_{0} \in M$

2. Gradient descent: $X_{i+1}=X_{i}-\nabla_{X} P\left(X^{\prime} \mid X\right)$

3. Enforce qualitative constraints: $X_{i+1} \in M$

4. Goto Step 2

\subsection{Initial Selection}

The algorithm requires the initial estimate to be a point-set residing on $M$. In this work, this is achieved this by randomly picking one point-set from the training data; however, other methods are possible. For instance, a linear combination of training-sets may yield an initial estimate closer to the solution. However, since $M$ is generally not a convex set, a linear combination of training-sets may not reside on $M$.

\subsection{Gradient Descent}

In order to refine an estimate, the algorithm will change the point-set according to the gradient of the posterior $\nabla_{X} P\left(X \mid X^{\prime}\right)$, which is simply the likelihood function. We use the sum of squared differences, as shown in equation 3 as an estimate of this function.

$$
\begin{aligned}
& \nabla_{X} P\left(X \mid X^{\prime}\right)=\lambda\left\|X-X^{\prime}\right\|^{2} \\
& 0<\lambda \leq 1
\end{aligned}
$$

A large $\lambda$ will result in a faster convergence, while the refined point-set may be pushed far away from $M$, making it harder to reproject. In this work we simply chose $\lambda=0.05$, without claiming it to be an optimal choice.

\subsection{Enforcing the Qualitative Constraints}

Given a set of points $X=p_{1}, \ldots, p_{n}$, and a constraints table $T$ encoding the manifold $M$, how do we update $X$ so that it complies with $T$ ? In order to visualize the complexity of the problem, note that a point-set changes its qualitative configuration as soon as one point crosses any line defined by two other points in the point-set. Assume that $\left[p_{1}, p_{3}, p_{4}\right]$ in figure 5 is a violating triplet with respect to $T$. Further, we want to fix this violation by moving point $p_{1}$ to the other side of line $\left[p_{3}, p_{4}\right]$. After this alteration, $p_{1}$ 


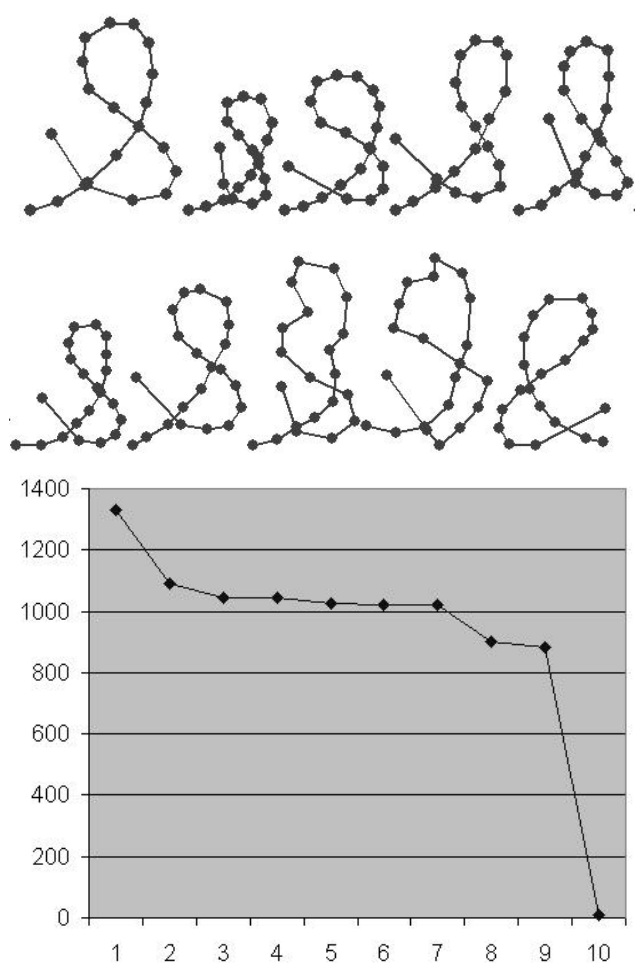

Fig. 4. The training shapes and the constraints plot for the ampersand

has also crossed lines $\left[p_{2}, p_{5}\right],\left[p_{3}, p_{5}\right]$ and $\left[p_{2}, p_{4}\right]$, which may cause new violations, depending on the constraints table. In this paper, we use the following heuristic that almost always resolves all violations. However, a perfect algorithm remains to be developed. Given a point-set $X$ and a constraints table $T$, our algorithm iterates over the following steps until all violations are resolved (or until no more violations can be resolved).

1. Select the point $p_{i} \in X$ that is a member of the largest number of violating triplets with respect to the constraint table.

2. For each violating triplet containing $p_{i}$, create a new point-set by moving $p_{i}$ to the other side of the line defined by the other two points in the triplet.

3. From all the new point-sets, keep the point-set with the least number of violating triplets.

In step 2 we refer to "The other side of the line". By this we mean a small distance, $\epsilon$, away from the line since that corresponds to the smallest alteration. However, optimization theory teaches us that in some cases, it is better to re-project further, in order to approach the center of the manifold, which we will explore in our future research. 


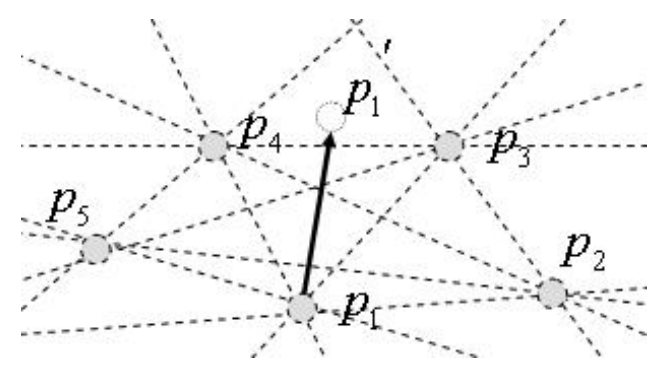

Fig. 5. Enforcing the qualitative constraints in a point-set

\subsection{Experimental examples of $2 D$ point-sets}

The algorithm was tested on a selection of point-sets. In this paper we show the results from point-sets representing hand-drawn characters and shapes, and point-sets representing sequences of human motion. For the characters, we manually drew a number of curves and spatially sampled them in order to generate point-sets, and correspondences between sets. In fig. 6 the training-set of a class of point-sets is shown in the first column. The second column shows a noisy point-set from the same class. The last column shows the result of the algorithm. For human motion We applied the algorithm

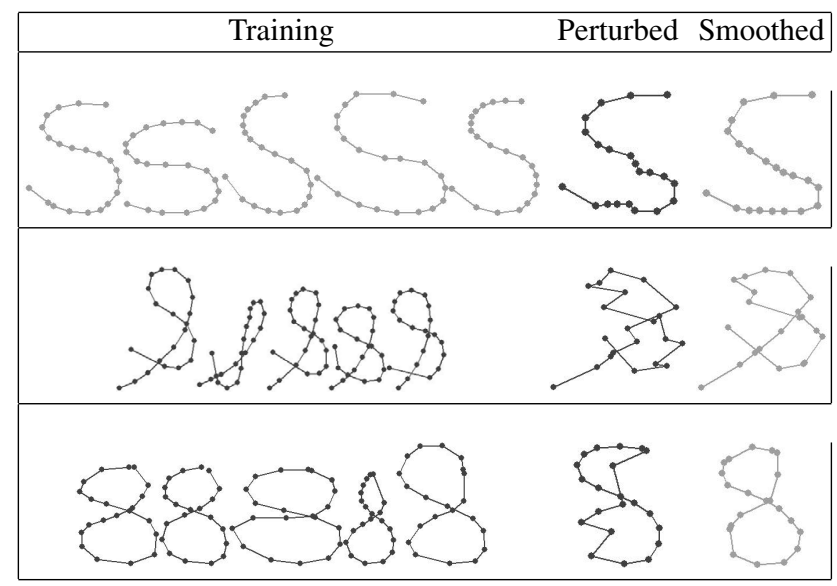

Fig. 6. The first column shows the training-shapes, used to learn the qualitative constraints. The second column shows a perturbed instance of a shape coming from this category. The last column shows the corrected perturbed shape.

to data from a number of sequences from athletics. When dealing with sequences, the point-set is generated by stacking the point-set of each time-frame into one tall matrix. This means that both temporal and structural constraints will be considered by the 
constraints-table. The results are illustrated in fig. 7. The first row shows an original shape, the second shows the same shape perturbed with noise. The row shows the corrected shape. The first two shapes are the same tennis-sequences perturbed with different amount of noise. The constraints-table was generated from 8 training sequences, and consisted of 12,296 rows (=constraining triplets). The first perturbed tennis-sequence contained 98 violations, primarily due to the right knee in frame 1, the right hand in frame 2 and the right foot in frame 3 . The second contained 918 violations, before being smoothed. The last column shows a weight-lift (snatch). The constraints-table for this was based on 5 training-sets, and contained 15984 rows. The perturbed point-set violated 2961 of these before being smoothed.

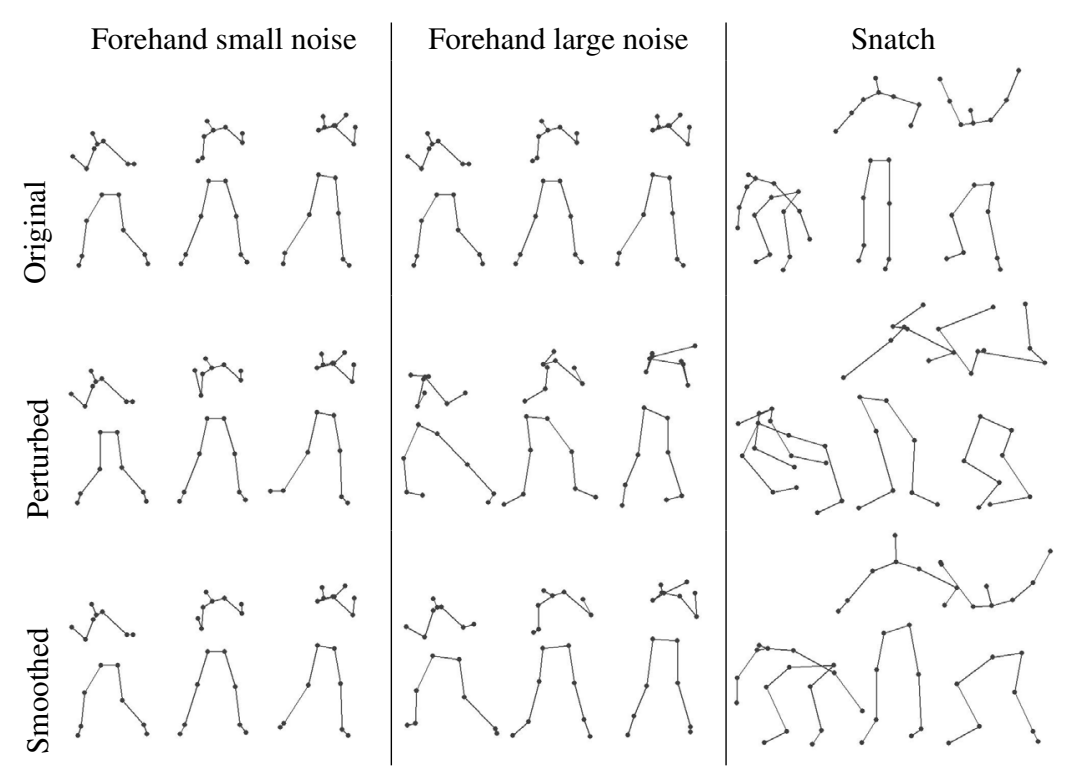

Fig. 7. The filtering algorithm applied to different 2D shapes and motions. The top row shows the original shape, the second row shows the perturbed shape, and the last row shows the restored shape.

\section{Conclusion and future work}

We have presented a method to make a rough estimate and representation of the manifold of possible events, and used it as prior information in the smoothing of point set data. By using this manifold as a prior constraint as opposed to trying to estimate the complete pdf, we avoid biasing our estimates towards the specific examples used in the learning set. It can be seen as a way of letting the measured data determine the smoothed estimate as far as possible, just using the priors to avoid qualitatively wrong 
answers. The method appears very promising on the notoriously difficult and important problem of estimating human motion data. In the near future, we will extend work to three dimensional human motion from monocular image sequences.

\section{References}

1. O. Aichholzer, F. Aurenhammer, and H. Krasser. Enumerating order types for small point sets with applications. In Proc. of the 17:th Ann. ACM Symp. Computational Geometry, pages 11-18, 2001.

2. O. Aichholzer, F. Aurenhammer, and H. Krasser. Points and combinatorics. Special Issue on Foundations of Information Processing of TELEMATIK, 1(7):12-17, 2002.

3. R. Bowden. Learning statistical models of human motion. In Proc. of the IEEE Workshop on Human Modeling, Analysis and Synthesis, CVPR, 2000.

4. M. Brand. Shadow puppetry. In Proceedings of the International Conference on Computer Vision, pages 1237-1244, 1999.

5. C. Bregler. Learning and recognizing human dynamics in video-sequences. In Proc. of the IEEE International Conference on Computer Vision and Pattern Recognition, pages 568-574, 1997.

6. Jos Gomes and Aleksandra Mojsilovic. A variational approach to recovering a manifold from sample points. In Proc. of the European Conference on Computer Vision, pages 3-17, 2002.

7. Michael E. Leventon and William T. Freeman. Bayesian estimation of $3 \mathrm{~d}$ human motion from an image sequence. Technical Report TR-98-06, MERL-A Mitsubishi Research Laboratory, 1998.

8. Vladimir Pavlovic, James M. Rehg, Tat-Jen Cham, and Kevin P. Murphy. A dynamic bayesian network approach to figure tracking using learned dynamic models. In Proc. of the IEEE International Conference on Computer Vision, volume 1, pages 94-101, 1999. 